\title{
The Cosmological Slingshot Scenario: a Stringy Proposal for the Early Time Cosmology
}

Cite as: AIP Conference Proceedings 1031, 172 (2008); https://

doi.org/10.1063/1.2972004

Published Online: 31 July 2008

Cristiano Germani, Nicolás Grandi and Alex Kehagias

Challenge us. What are your needs for periodic signal detection?

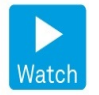

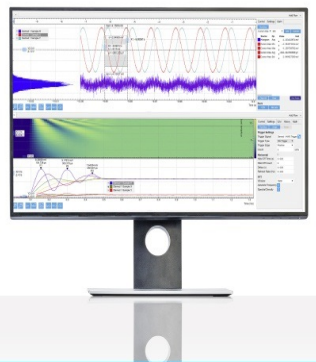

Zurich Instruments 


\title{
The Cosmological Slingshot Scenario: a Stringy Proposal for the Early Time Cosmology ${ }^{1}$
}

\author{
Cristiano Germani*, Nicolás Grandi ${ }^{\dagger}$ and Alex Kehagias** \\ ${ }^{*}$ SISSA and INFN - via Beirut 4, 34014 Trieste, Italy \\ ${ }^{\dagger}$ IFLP, CCT La Plata, CONICET - Dto. de Física, UNLP \\ Casilla de Correos 67, CP1900 La Plata, Argentina \\ ** Department of Physics, National Technical University of Athens \\ GR-15773, Zografou, Athens, Greece
}

\begin{abstract}
In The Cosmological Slingshot Scenario, our Universe is a D3-brane that extends in the $4 d$ noncompact directions of a warped Calabi-Yau compactification of IIB Supergravity. Early time cosmology corresponds to a period in which the brane moves inside a warped throat, a nonvanishing angular momentum ensuring that the trajectory of the brane has a turning point. The corresponding induced metric on the $D 3$-brane experiences a cosmological evolution with a bounce. In this framework, the homogeneity, flatness, and isotropy problems of standard cosmology might be avoided. The power spectrum of primordial perturbations of the brane embedding can be found and it is shown to be in agreement to WMAP data.
\end{abstract}

Keywords: String Theory, D-branes, Mirage Cosmology.

PACS: $11.25 . \mathrm{Wx}, 98.80 .-\mathrm{k}$

\section{INTRODUCTION}

The Cosmological Slingshot Scenario [1, 2, 3] is a proposal for the cosmic earlytime evolution in the String Theory context. According to that, our Universe is a $D 3$ brane moving in a String Theory background of the form $\mathscr{M}^{4} \times K^{6} . \mathscr{M}^{4}$ is a warped Minkowskian space-time and $K^{6}$ is a compact Calabi-Yau (CY) space. The latter includes a throat sourced by a stack of a large number $(N)$ of other D3-branes. The Slingshot is characterized by a non-trivial orbital motion of the Universe in the compact space around the stack of D3-branes. If back-reaction can be neglected (probe brane approximation), a brane observer measures a $4 d$ metric induced in terms of the brane embedding, that defines a cosmological brane evolution commonly called Mirage Cosmology [4].

The early-time evolution (i.e. well before nucleosynthesis) corresponds to the motion of the D3-brane deep into the throat (Slingshot era) moving towards the hat of the compact space. Since $N$ is taken to be large, close to the stack the probe brane approximation can be used. The late-time cosmology starts when the D3-brane reaches the hat of the $\mathrm{CY}$, the probe brane approximation breaks down and local gravity à la RandallSundrum [5,6] dominates the cosmological evolution. Under this approximation, the

\footnotetext{
${ }^{1}$ Based on [1] and [2].
} 
Slingshot brane observer experiences a non-singular bouncing cosmology. Additionally, as we shall show later on, the Standard Cosmology problems (i.e. homogeneity, isotropy and flatness) might be avoided in the brane induced cosmology.

\section{THE COSMOLOGICAL SLINGSHOT SCENARIO}

\section{Setup}

To make the discussion concrete, we will consider a probe D3-brane moving in a throat of a Calabi-Yau (CY) compact manifold, whose metric and Ramond-Ramond 5form can be written as

$$
\left.d s^{2}=h^{-\frac{1}{2}} d s_{\mathbb{M}_{4}}^{2}+h^{\frac{1}{2}}\left(d r^{2}+r^{2} d s_{5}^{2}\right)\right), \quad C_{0123}=1-\frac{1}{h},
$$

where $h$ is a function of $r$ only, and $d s_{5}^{2}$ is the base manifold characterizing the remaining part of the transverse space.

The dynamics of a probe brane is governed by the Dirac-Born-Infeld action with the Wess-Zumino coupling

$$
S_{D B I}+S_{W Z}=-T_{3} \int \sqrt{-g_{i}} d^{4} \xi-T_{3} \int C_{(4)} .
$$

We assume that all other fields on the brane are switched off and matter is created later. The sign of the Wess-Zumino term has been chosen to represent a D3-brane and $T_{3}=1 /(2 \pi)^{3} g_{s} l_{s}^{4}$ is the tension of the probe. The probe brane is extended along the $\mathscr{M}_{4}$ directions, so that it looks like a point particle moving in the transverse space. In the static gauge the resulting induced metric is

$$
d s_{i}^{2}=h^{-1 / 2}\left[-\left(1-h\left(r^{\prime 2}+r^{2} \Omega_{5}^{\prime 2}\right)\right) d t^{2}+d \vec{x} \cdot d \vec{x}\right],
$$

where a prime $\left({ }^{\prime}\right)$ denotes a derivative with respect to $t$ and we have assumed that the only non-vanishing transverse momenta are in the $r, \Omega_{5}$ directions, $\Omega_{5}$ representing an angle in the transverse space. Replacing this induced metric into the brane action (2) we get

$$
S=-T_{3} V_{3} \int d t\left[\frac{1}{h} \sqrt{1-h\left(r^{\prime 2}+r^{2} \Omega_{5}^{\prime 2}\right)}+1-\frac{1}{h}\right],
$$

where $V_{3}$ is the un-warped volume of the directions parallel to the probe. The resulting equations of motion have first integrals provided by

$$
U=\frac{1}{h}\left[\frac{1}{\sqrt{1-h\left(r^{\prime 2}+r^{2} \Omega_{5}^{\prime 2}\right)}}-1\right], \quad J=\frac{r^{2}}{\sqrt{1-h\left(r^{\prime 2}+r^{2} \Omega_{5}^{\prime 2}\right)}} \Omega_{5}^{\prime},
$$


that can be inverted to get

$$
r^{\prime 2}=\frac{1}{(1+h U)^{2}}\left(2 U-\frac{J^{2}}{r^{2}}+h U^{2}\right) .
$$

The motion will take place at the values of $r$ that make this expression positive. Moreover wherever the expression in parenthesis vanishes, the trajectory will have a turning point.

To interpret the induced metric (3) as the cosmology experienced by an observer on the brane, we need to define the cosmic time according to

$$
d \tau=h^{-1 / 4} \sqrt{1-h\left(r^{\prime 2}+r^{2} \Omega_{5}^{\prime 2}\right)} d t
$$

in terms of which the metric (3) is identified as a Friedman-Robertson-Walker metric with scale factor

$$
a=h^{-1 / 4}(r) \text {. }
$$

When $r(t)$ has a turning point, it is easy to see that the same happens to $a(\tau)$, generating a nonsingular bouncing cosmology. A Friedmann equation can be written for such cosmology by changing to cosmic time in Eq.(6) and dividing the result by $a^{2}$

$$
H^{2}=\frac{1}{16} a^{6} h_{r}^{2}(a)\left[2 U-\frac{J^{2}}{r^{2}(a)}+\frac{U^{2}}{a^{4}}\right],
$$

where $H=\dot{a} / a$ is the (mirage) Hubble constant (here $\dot{a}=\partial_{\tau} a$ ).

The model is completed by smoothly pasting this Mirage era to a local gravity driven late evolution when the brane reaches the top of the CY and gravity becomes localized á la Randall-Sundrum [5,6]. There, the standard late time evolution of the observed Universe is supposed to be well reproduced by the brane dynamics. This assumption involves a transition from a mirage dominated era with a moving brane without any matter, into a local gravity dominated era with an static brane and matter fields excited on it. This transition has to be understood as an analogous of the reheating process in standard inflationary models. It entails a dynamical mechanism under which the kinetic energy of the brane is passed to matter fields. The description of this dynamics as well as the robustness of our predictions for physical observables is an open point that is left for future research.

A concrete example of the above proposed situation is given by a probe moving in the $\mathrm{AdS}_{5} \times \mathrm{S}_{5}$ background. In it, the metric takes the form (1) with

$$
h_{\mathrm{AdS}}=\frac{L^{4}}{r^{4}}, \quad L^{4}=4 \pi l_{s}^{4} N g_{s},
$$

where $l_{s}$ is the string length and $g_{s}$ is the string coupling. The supergravity approximation is valid as long as the curvature radius of the solution is large compared to the string length $l_{s}$. String perturbation theory on the other hand requires $g_{s} \ll 1$.

A close look to Eq.(6) shows that, in the present case, the second factor is a quadratic function of the variable $r^{-2}$, that will have a root as long as its discriminant is positive 
$J^{4}-8 U^{3} L^{4}>0$. For values of $r$ larger that that of the root the function is positive. Then whenever this inequality is satisfied, the probe orbit has an inner turning point.

The resulting scale factor (8) reads

$$
a_{\mathrm{AdS}}=\frac{r}{L} .
$$

In this background, the induced Friedmann equation (9) becomes

$$
H_{\mathrm{AdS}}^{2}=\frac{1}{L^{2}}\left[\frac{2 U}{a_{\mathrm{AdS}}^{4}}-\frac{(J / L)^{2}}{a_{\mathrm{AdS}}^{6}}+\frac{U^{2}}{a_{\mathrm{AdS}}^{8}}\right],
$$

where $H_{\mathrm{AdS}}=\dot{a}_{\mathrm{AdS}} / a_{\mathrm{AdS}}$ is the (mirage) Hubble constant. Since when $J^{4}-8 U^{3} L^{4}>0$ the orbit has a turning point, in that case the corresponding cosmology has a bounce.

Another example is that of a probe brane motion in a Klebanov-Strassler (KS) throat [7]. In the region far from the tip of the throat, KS geometry can be well approximated by the Klebanov-Tseytlin (KT) metric [8] that takes the form (5). The warp factor reads

$$
h_{\mathrm{KS}}=\frac{L^{4}}{r^{4}} \ln \left(\frac{r}{r_{s}}\right), \quad L^{2}=\frac{9}{\sqrt{8}} l_{s}^{2} M g_{s},
$$

where $r_{s}$ is proportional to the radius of the blown up sphere at the tip of the cone. To trust the KT approximation we need to ensure that the probe brane will never reach $r \simeq r_{s}$. This will be the case iff $r_{s}$ lies inside the forbidden region $r^{\prime 2}<0$. Going back to equation (6) and evaluating it at $r_{s}$, we see that this is true when $2 r_{s} U-J^{2}<0$, the probe motion having a turning point at some value of $r$ larger than $r_{s}$.

The resulting scale factor is

$$
a_{\mathrm{KS}}=\frac{r}{L} \ln ^{-1 / 4}\left(\frac{r}{r_{s}}\right),
$$

and, under the assumption $2 r_{S} U-J^{2}<0$, it corresponds to a bouncing cosmology.

An important ingredient in our argument is that the scale factor for a brane moving in a KS throat (14) can be rewritten as a conformal re-scaling of the corresponding scale factor for a brane moving in $\mathrm{AdS}_{5}$. Indeed, when written in conformal time, the induced metric on the brane reads $d s_{i}^{2}=a^{-2} d s_{\mathbb{N}_{4}}^{2}$ and we can write

$$
a_{\mathrm{KS}}=\Omega\left(a_{\mathrm{AdS}}\right) a_{\mathrm{AdS}}, \quad \text { with } \Omega\left(a_{\mathrm{AdS}}\right)=\log ^{-1 / 4}\left(a_{\mathrm{AdS}} \frac{L}{r_{s}}\right) .
$$

It should be kept in mind that our approximations are valid whenever $a_{\mathrm{AdS}} \gg r_{S} / L$. Under such a re-scaling, the Hubble constant changes as

$$
H_{\mathrm{KS}}=\left(1+a_{\mathrm{AdS}} \frac{d \ln \Omega}{d a_{\mathrm{AdS}}}\right) H_{\mathrm{AdS}} .
$$




\section{The Problems of Standard non-Inflationary Cosmology}

We are now ready to study how standard cosmological problems are solved in the Slingshot scenario.

A. Homogeneity. As explained above, in both the AdS case and the KS throat the probe brane experiences a bounce in the String frame. This immediately ensures that homogeneity problem is solved. To check this explicitly, we write the co-moving horizon

$$
\Delta \eta=\int_{\eta_{i}}^{\eta_{0}} d \eta
$$

where $\eta$ is conformal time and $\eta_{i}$ is its smallest value. To solve homogeneity problem it is required that $\Delta \eta>H_{0}^{-1}$. Since we have $\eta_{i} \rightarrow-\infty$ due to the absence of a cosmological singularity, this condition is trivially satisfied.

B. Isotropy. In the AdS case, mirage matter contributes to Friedmann equation (12) with a term $\rho \sim a_{\text {AdS }}^{-8}$. This term dominates over the shear $\rho_{\text {shear }} \sim a_{\text {AdS }}^{-6}$ at early times, avoiding the chaotic behavior [9]. To check whether this is true in the KS case, we should verify that the corresponding mirage contribution dominates over the shear. The form of this contribution can be read from (16), and we can write the quotient

$$
\sqrt{\frac{\rho_{\text {shear }}}{\rho}} \propto\left(1+a_{\mathrm{AdS}} \frac{d \ln \Omega}{d a_{\mathrm{AdS}}}\right)^{-1} \frac{a_{\mathrm{AdS}}}{\Omega^{3}} .
$$

The proportionality constant in (18) parameterizes the anisotropic perturbations in the pre-bounce era. It is simple to check that (18) is an increasing function of $a_{\text {AdS }}$ in the region $a \gtrsim e^{((2 \sqrt{2}-1) / 4)} r_{s} / L \simeq 1.57 r_{s} / L$. As we assumed that the Slingshot brane never approaches the tip of the KS throat, this condition is automatically satisfied. Therefore, $\rho_{\text {shear }} / \rho$ decreases very rapidly close to the bouncing point in the pre-bounce era, solving the isotropy problem.

C. Flatness. The curvature contribution to the Hubble equation ${ }^{2}$ can be disregarded if the quantity $\left|\Omega_{\text {Total }}-1\right|=1 / a^{2} H^{2}$ passes through a minimum where it satisfies the phenomenological constraint

$$
\left|\Omega_{\text {Total }}-1\right|_{\min }<10^{-8} .
$$

For the AdS case, the above quantity evaluated at its minimum reads

$$
\begin{aligned}
\left|\Omega_{\text {Total }}-1\right|_{\min } & =\frac{\left(J^{2}+\sqrt{J^{4}-6 L^{4} U^{3}}\right)^{3}}{4 L^{2} U^{2}\left(J^{4}-4 L^{4} U^{3}+J^{2} \sqrt{J^{4}-6 L^{4} U^{3}}\right)} \\
& \simeq\left(\frac{J}{2 L U}\right)^{2}+\mathscr{O}\left(\frac{L^{4} U^{3}}{J^{4}}\right)<10^{-8} .
\end{aligned}
$$

\footnotetext{
2 The details of how to include a curvature term in the mirage Hubble equation can be found in [1].
} 
This condition is not a fine tuning in parameter space, but just a restriction to a two dimensional region. In this sense flatness problem might be alleviated in the Slingshot scenario.

For the KS case we have, after conformal re-scaling

$$
\left|\Omega_{\text {Total }}-1\right|=\frac{f^{2}}{a_{\text {AdS }}^{2} H_{\text {AdS }}^{2}}, \quad f=\frac{4 \ln \left(a_{\mathrm{AdS}} L / r_{S}\right)}{4 \ln \left(a_{\mathrm{AdS}} L / r_{S}\right)-1} .
$$

The KT approximation is valid for $r_{\min } \gg r_{s}$; to fix ideas we will use $r_{\min }>10^{2} r_{s}$. In this region we have $f=\mathscr{O}(1)$ and decreasing in $a_{\text {AdS }}$. Consequently, the flatness problem in the KS space might, in good approximation, be alleviated by the same choice of parameters used in the AdS case.

\section{PRIMORDIAL PERTURBATIONS}

\section{The Hollands-Wald Mechanism}

In inflationary scenarios the primordial perturbations are produced by quantum fluctuations of the inflaton field and are codified into its two point correlation function in the vacuum state. However, these fluctuations are over-damped by the expansion of the Universe at super-horizon scales. At these scales then, the quantum state becomes characterized by a large occupation number and the system collapses into a classical state. This classical state represents a random spectrum of perturbations whose variance is given by the quantum correlations evaluated at the quantum-to-classical transition point [10].

Let us now turn our attention into the mechanism proposed by Hollands and Wald in [11]. A perturbation of wavelength $\lambda$ smaller than a typical quantum scale, say $l_{c}$, is in its quantum vacuum. In an expanding background, the wavelength of a perturbation grows in time $(\lambda \propto a)$ and whenever $\lambda \sim l_{c}$, or in other words, as soon as the perturbation becomes macroscopic, wavelengths bigger than the horizon scale collapse into a classical random state. In the proposal of [11], the relevant fluctuations are so continuously "created" at "super-horizon" scales. Thus, a coherent spectrum of classical perturbations is produced with variance given by matching the classical correlations with the quantum correlations at the quantum-to-classical transition point.

It has been suggested [12] that a space-time uncertainty relation $\Delta X \Delta T \gtrsim l_{s}^{2}$ should be realized in String Theory, $\Delta X$ and $\Delta T$ representing the uncertainties in measuring space and time distances. Since the smallest length that can be probed in String Theory is the 11-dimensional Planck length, $\Delta X>l_{P^{11}} \sim g_{s}^{1 / 3} l_{s}$, we obtain that the smallest measurable time is $\Delta T \gtrsim g_{s}^{-1 / 3} l_{s}$. The period of a wave propagating in a $D$-brane is $2 \pi \omega^{-1} \sim \lambda$ and cannot be smaller than that $\Delta T$, implying

$$
\lambda>l_{s} g_{s}^{-1 / 3} .
$$

We therefore have a minimal wavelength for a perturbation on the brane as in the Hollands-Wald mechanism. This strongly suggests to use such mechanism to study the 
cosmological perturbations in the Slingshot model $^{3,4}$.

Technically, the mechanism explained above introduces a vacuum state in which the perturbation is destroyed (coming from the pre-bounce era) and then created again (after the bounce) at the time $\eta_{*}$ in which the proper wavelength of the corresponding quantum mode reaches the value

$$
a\left(\eta_{*}\right) / k \equiv a_{*} / k=l_{c} .
$$

We start by perturbing the embedding of the probe brane by writing $r=r(\eta)+$ $\delta r(\eta, \vec{x})$ and $\Omega_{5}=\Omega_{5}(\eta)+\delta \Omega(\eta, \vec{x})$, where $r(\eta), \Omega_{5}(\eta)$ are the solutions of the equations of motion obtained from action (2), written as functions of the conformal time $\eta$. In the non-relativistic approximation $h U \ll 1$ we have $\eta \equiv t$ and Eqs.(5) are integrated to

$$
r(\eta)=\sqrt{2 U \eta^{2}+\frac{J^{2}}{2 U}}, \quad \Omega_{5}(\eta)=\arctan \left(\frac{2 U}{J} \eta\right) .
$$

Note that we have a turning point at $r_{\min }=J / \sqrt{2 U}$.

In what follows, we will use as our variable the Bardeen potential $\delta \Phi_{k}=\delta r_{k} / r$ [15], in terms of which the action (2) can be expanded to quadratic order in $\delta$ 's and their derivatives, getting (in Fourier space)

$$
S=T_{3} \sum_{k} \int d \eta\left(\frac{r^{2}}{2}\left(\delta \Phi_{k}^{\prime 2}+\delta \Omega_{k}^{\prime 2}-k^{2}\left(\delta \Phi_{k}^{2}+\delta \Omega_{k}^{2}\right)\right)+J \delta \Omega_{k}^{\prime} \delta \Phi_{k}-J \delta \Omega_{k} \delta \Phi_{k}^{\prime}\right) .
$$

\section{Power Spectrum and Spectral Index}

The canonical quantization procedure applied to the action (25) provide the normalized operators

$$
\delta \hat{\Phi}_{k}=u_{1} \hat{a}_{1}+u_{2} \hat{a}_{2}+c . c ., \quad \delta \hat{\Omega}_{k}=v_{1} \hat{a}_{1}+v_{2} \hat{a}_{2}+c . c .,
$$

where $a_{i}, a_{i}^{\dagger}$ are standard annihilation and creation operators, and

$$
\begin{aligned}
u_{1} & =\sqrt{\frac{U}{k T_{3}}} \frac{\eta}{r^{2}} e^{-i k \eta}, & u_{2} & =\sqrt{\frac{1}{U k T_{3}}} \frac{J}{2 r^{2}} e^{-i k \eta}, \\
v_{1} & =u_{2}=\sqrt{\frac{1}{U k T_{3}}} \frac{J}{2 r^{2}} e^{-i k \eta}, & v_{2} & =-u_{1}=-\sqrt{\frac{U}{k T_{3}}} \frac{\eta}{r^{2}} e^{-i k \eta} .
\end{aligned}
$$

\footnotetext{
${ }^{3}$ In fact, the complete spectrum will include a blue part [13] coming from the perturbations created in the pre-bounce era, that were not contracted up to the minimal wavelength at the bounce, and an additional red part [3] due to the ones that were. Here we will fix out attention in this second part

${ }^{4}$ In the original proposal of [11] the perturbation was produced by the same radiation which sets the CMB. However, as pointed out by [14], the perturbation coming out from the horizon today, was necessarily born when the energy density of radiation was much bigger than the Planck energy, which makes the mechanism unreliable. In the Slingshot instead, perturbations are created by brane fluctuations in a regime in which the supergravity approximation is still valid, and no extra quantum effect takes place. Moreover, super-horizon causality is not required, since in the Slingshot perturbations are overdamped at sub-horizon scales $k<J / r^{2}$.
} 
We are interested in the correlation of the Bardeen potential $\delta \hat{\Phi}$ at the time of creation $\eta_{*}$. Using the above formulas to define $r_{*}=r\left(\eta_{*}\right)$, it is straightforward to check that

$$
\left\langle\delta \hat{\Phi}_{k} \delta \hat{\Phi}_{k^{\prime}}\right\rangle=\delta_{k, k^{\prime}} \frac{1}{2 k T_{3} r_{*}^{2}} .
$$

We will consider the transition point of the quantum to the classical description in the region in which $k \ll J / r^{2}$. In this limit, we can discard the $k^{2}$ term in the action (25) and write its classical solutions as

$$
\delta \Phi_{k}=\frac{C_{k}}{2 J}+A_{k} \sin \left(2 \theta+\phi_{k}\right), \quad \delta \Omega=-\frac{D_{k}}{2 J}+A_{k} \cos \left(2 \theta+\phi_{k}\right),
$$

where $\theta=\Omega_{5}(\eta)-\Omega_{5}\left(\eta_{*}\right)$ and $\phi_{k}, C_{k}, D_{k}, A_{k}$ are constants of integration, that can be written as

$$
\begin{aligned}
& C_{k}=r^{2} \delta \Omega_{k}^{\prime}+2 J \delta \Phi_{k}, \quad D_{k}=r^{2} \delta \Phi_{k}^{\prime}-2 J \delta \Omega_{k}, \\
& A_{k}=\frac{r^{2}}{2 J}\left[\delta \Phi_{k}^{\prime} \cos \left(2 \theta+\phi_{k}\right)-\delta \Omega_{k}^{\prime} \sin \left(2 \theta+\phi_{k}\right)\right] .
\end{aligned}
$$

We now consider initial conditions arising from the matching of the classical to the quantum system at the time $\eta=\eta_{*}$. Therefore $C_{k}, D_{k}, A_{k}$ will be taken as Gaussian stochastic variables with correlations $\langle\ldots\rangle_{c}$ matching the quantum correlators $\langle\ldots\rangle$ at $\eta=\eta_{*}$.

Using the quantum solutions described above at the matching point $\eta=\eta_{*}$ after a lengthly but straightforward calculation we have

$$
\begin{aligned}
\left\langle C_{k} C_{k^{\prime}}\right\rangle_{c} & =\delta_{k, k^{\prime}} \frac{k^{2} r_{*}^{2}+2 U}{2 k T}, \quad\left\langle A_{k} A_{k^{\prime}}\right\rangle_{c}=\delta_{k, k^{\prime}} \frac{k^{2} r_{*}^{2}+2 U}{8 J^{2} k T}, \\
\left\langle A_{k} C_{k^{\prime}}\right\rangle_{c} & =\delta_{k, k^{\prime}} \frac{\sin \phi}{4 k T J r_{*}^{2}}\left\{2 J^{2}-2 U r_{*}^{2}-k^{2} r_{*}^{4}-4 J U \eta_{*} \cot \phi_{k}\right\} .
\end{aligned}
$$

The matching of

$$
\left\langle\delta \Phi_{k} \delta \Phi_{k^{\prime}}\right\rangle_{c}=\left\langle\delta \hat{\Phi}_{k} \delta \hat{\Phi}_{k^{\prime}}\right\rangle,
$$

requires $\phi_{k}=\pi / 2$; this is the selection of positive frequencies.

In general correlators depend on time through $\theta$. However in the region $k \ll J / r^{2}$, the oscillation rapidly stabilizes in time when $2 U \eta_{\text {asymp. }}>2 \pi J$. We will consider this to happen well before the nucleosynthesis. At this time then

$$
\delta \Phi_{k}=\frac{C_{k}}{2 J}-A_{k} \cos \left(2 \Omega_{5}\left(\eta_{*}\right)\right)=\frac{C_{k}}{2 J}+A_{k}\left(1-\frac{2 r_{\min }^{2}}{r_{*}^{2}}\right) .
$$

Using the initial conditions found above we then get in the limit $k \ll J / r_{*}^{2}<J / r_{\min }^{2}$

$$
\left.\left\langle\delta \Phi_{k} \delta \Phi_{k^{\prime}}\right\rangle_{c}\right|_{\eta>\eta_{\text {asymp. }}} \simeq \frac{\delta_{k, k^{\prime}}}{2 k T r_{*}^{2}}\left[1-\left(\frac{r_{\min }}{r_{*}}\right)^{2}\right]
$$


so the power spectrum of temperature fluctuations is

$$
P(k) \simeq \frac{1}{2 k T_{3} r_{*}^{2}}\left[1-\left(\frac{r_{\min }}{r_{*}}\right)^{2}\right] .
$$

A consistency condition for the production of the perturbation is that $r_{\min }<r_{*}$. So we see that in the limit $r_{\min } \ll r_{*}$ we obtain the power spectrum introduced in [1].

Since we assumed that a perturbation is created when its physical wavelength reaches a fixed value $l_{c}$, we have from Eq.(23), $k l_{c}=a_{*}$. In the AdS metric Eq.(11) implies $k l_{c} L=r_{*}$, resulting in the power spectrum

$$
P(k) \simeq \frac{1}{2 T_{3}\left(l_{c} L\right)^{2} k^{3}}\left[1-\frac{r_{\min }^{2}}{\left(l_{c} L\right)^{2} k^{2}}\right],
$$

for which the scalar spectral index $n_{s}-1=d \ln \left(k^{3} P(k)\right) / d \ln k$ reads

$$
n_{s} \simeq 1-\frac{2}{1-\frac{\left(l_{c} L\right)^{2}}{r_{\min }^{2}} k^{2}},
$$

and we see that the flat spectrum found in [1] at the matching point is blue-shifted by the subsequent time evolution.

In the Klebanov-Tseytlin (KT) metric on the other hand, the condition $k l_{c}=a_{*}$ is solved by $r_{*}=r_{s} e^{-W_{-1}(-\zeta) / 4}$ where $\zeta=4\left(r_{s} / L l_{c} k\right)^{4} \leq e^{-1}$ and $W_{-1}(x)$ is the negative branch of Lambert's $W$-function. Then the power spectrum (36) is explicitly written as

$$
P(k)=\frac{1}{2 T_{3} k r_{s}^{2}} e^{\frac{1}{2} W_{-1}(-\zeta)}\left(1-\left(\frac{r_{\min }}{r_{s}}\right)^{2} e^{\frac{1}{2} W_{-1}(-\zeta)}\right),
$$

whereas the scalar spectral index turns out to be

$$
\begin{aligned}
n_{s} & =1+\frac{2}{1+W_{-1}(-\zeta)}\left(1-\frac{W_{-1}(-\zeta)}{1-\left(r_{s} / r_{\text {min }}\right)^{2} e^{-\frac{1}{2} W_{-1}(-\zeta)}}\right) \\
& \simeq 1+\frac{2}{\ln (\zeta)}-\frac{2 \sqrt{\zeta}}{\sqrt{\zeta}-\left(r_{s} / r_{\text {min }}\right)^{2}}
\end{aligned}
$$

where the expansion of the Lambert $\mathrm{W}$ function for small argument $W(-\zeta) \simeq \ln (\zeta)+\cdots$ was used in the second line. Since in this limit $\ln (\zeta)<0$, the first correction on $n_{s}$ is negative. On the other hand, the second correction is red or blue according to the sign of its denominator. It will be negative whenever

$$
\sqrt{\zeta}>r_{s}^{2} / r_{\min }^{2}
$$

from which we immediately see that long wavelengths are red-shifted. 
On the other hand, if the last term is positive, then $\sqrt{\zeta}<r_{s}^{2} / r_{\min }^{2}$ and the overall sign of the correction has to be evaluated taking into account the joint contribution of both terms in (40). After some manipulations we find that the correction is red whenever

$$
\sqrt{\zeta}(1-2 \log \sqrt{\zeta})<\left(r_{s} / r_{\min }\right)^{2}
$$

from which we conclude that short wavelengths are also red-shifted, and there is an intermediate range of wavelengths that is blue-shifted.

\section{BACK-REACTION AND EFFECTIVE 4D THEORY}

The 4D effective theory for warped compactifications of IIB supergravity with (static) D-branes has been derived by a perturbative approach in [16], and by a gradient expansion method in [17]. Using these results, in [2] the following effective 4d Lagrangian describing Slingshot cosmology has been found

$$
S_{\text {brane }}=\frac{L^{2}}{2} \int d^{4} x \sqrt{-g}\left[\left(\frac{1}{\kappa^{2} r^{2}}-\frac{T_{3}}{6 N}\right) R+\frac{6}{\kappa^{2}} \frac{(\nabla r)^{2}}{r^{4}}-\frac{T_{3}}{N}\left(\nabla \Omega_{5}\right)^{2}\right] .
$$

The resulting equations of motion, specialized to a Friedmann-Robertson-Walker background with scale parameter $a(\eta)$, result into the following set of equations

$$
\begin{aligned}
& \frac{r^{\prime \prime}}{r}+2 \frac{r^{\prime}}{r}\left(\frac{a^{\prime}}{a}-\frac{r^{\prime}}{r}\right)+\Omega_{5}^{\prime 2}=0, \quad \frac{d}{d \eta}\left(a^{2} \Omega_{5}^{\prime}\right)=0, \\
& \frac{T_{3} \kappa^{2}}{N}\left(\frac{a^{\prime 2}}{a^{2}}+\Omega_{5}^{\prime 2}\right)=\frac{6}{r^{2}}\left(\frac{a^{\prime}}{a}-\frac{r^{\prime}}{r}\right)^{2} .
\end{aligned}
$$

As can be checked by direct substitution, an exact solution of the full system is

$$
a=\frac{1}{L} \sqrt{\frac{J^{2}}{2 U}+2 U \eta^{2}}, \quad \Omega_{5}^{\prime}=\frac{J}{L^{2}} \frac{1}{a^{2}}, \quad r=\frac{a L}{1+\kappa \sqrt{\frac{U T_{3}}{3 N} \eta}} .
$$

We obtained the same scale factor evolution as in the mirage approximation in [2], but now considering local gravity back-reactions. The only difference from the mirage approximation is on the $r$ evolution, due to the denominator $1+\kappa \sqrt{U T_{3} / 3 N} \eta$. Since $\kappa \sqrt{U T_{3} / 3 N}$ is supposed to be small, the mirage approximation breaks down at very late or very early times, i.e. when the brane leaves the throat, as expected. When this denominator is taken into account, the system is no longer time symmetric and there is a field singularity at the time in which the denominator vanishes. However, since the extradimensional space is compact $r<r_{\max }$, where $r_{\max }$ defines the cut-off of the compact space, this singularity is just fictitious, and the effective action cannot be trusted when $1+\kappa \sqrt{U T_{3} / 3 N} \eta \simeq 0$. There a description à la Randall-Sundrum [6] must be used. 


\section{SUMMARY}

The Cosmological Slingshot scenario provides an example of a bouncing cosmology in which the dynamics of the bounce is under control. It provides alternative solutions to the problems of standard cosmology, and the resulting perturbations spectrum is in agreement with WMAP data. In the way it has been presented here it still has to be checked that all the constraints, that appeared during our calculations due to our approximations and to phenomenological inputs, are mutually compatible. Even if in [1] some of these cross checks have been performed successfully, a complete cross-checking is still needed, and it may be the case that all the problems of standard cosmology cannot be solved at the same time. For example, to make our solution of flatness problem compatible with perturbation spectrum, a very strong lower bound on the conserved quantity $U$ is needed. The model is currently under research.

\section{ACKNOWLEDGEMENTS}

C.G. wishes to thank Cliff Burgess and Toni Riotto for useful discussions. This work is partially supported by the European Research Training Network MRTN-CT-2004005104 and the PEVE-NTUA-2007/10079 programme. N.E.G. wants to thank Department of Particle Physics of Santiago de Compostela University for hospitality during part of this work.

\section{REFERENCES}

1. C. Germani, N. E. Grandi and A. Kehagias, "A stringy alternative to inflation: The cosmological slingshot scenario," Class. Quant. Grav. in press (2008) [arXiv:hep-th/0611246].

2. C. Germani, N. Grandi and A. Kehagias, "The Cosmological Slingshot Scenario: Myths and Facts," arXiv:0706.0023 [hep-th].

3. C. Germani and M. Liguori, "Matching WMAP 3-yrs results with the Cosmological Slingshot Primordial Spectrum,” arXiv:0706.0025 [astro-ph].

4. A. Kehagias and E. Kiritsis, "Mirage cosmology," JHEP 9911, 022 (1999) [arXiv:hep-th/9910174].

5. L. Randall and R. Sundrum, "An alternative to compactification," Phys. Rev. Lett. 83, 4690 (1999) [arXiv:hep-th/9906064].

6. T. Shiromizu, K. i. Maeda and M. Sasaki, "The Einstein equations on the 3-brane world," Phys. Rev. D 62, 024012 (2000) [arXiv:gr-qc/9910076].

7. I. R. Klebanov and M. J. Strassler, "Supergravity and confining gauge theory: Duality cascades and chiSB-resolution of naked singularities," JHEP 0008, 052 (2000) [arXiv:hep-th/0007191].

8. I. R. Klebanov and A. A. Tseytlin, "Gravity duals of supersymmetric SU(N) x SU(N+M) gauge theories," Nucl. Phys. B 578, 123 (2000) [arXiv:hep-th/0002159].

9. J. K. Erickson, D. H. Wesley, P. J. Steinhardt and N. Turok, "Kasner and mixmaster behavior in universes with equation of state w >= 1," Phys. Rev. D 69, 063514 (2004) [arXiv:hep-th/0312009]; D. H. Wesley, P. J. Steinhardt and N. Turok, "Controlling chaos through compactification in cosmological models with a collapsing phase," Phys. Rev. D 72, 063513 (2005) [arXiv:hep-th/0502108].

10. A. R. Liddle and D. H. Lyth, Cosmological inflation and large-scale structure, Cambridge University Press, United Kingdom, 2000.

11. S. Hollands and R. M. Wald, "An alternative to inflation," Gen. Rel. Grav. 34, 2043 (2002) [arXiv:grqc/0205058];

S. Hollands and R. M. Wald, "Comment on inflation and alternative cosmology," arXiv:hepth/0210001. 
12. M. Li and T. Yoneya, "D-particle dynamics and the space-time uncertainty relation," Phys. Rev. Lett. 78, 1219 (1997) [arXiv:hep-th/9611072].

13. R. Brandenberger, H. Firouzjahi and O. Saremi, "Cosmological Perturbations on a Bouncing Brane," JCAP 0711, 028 (2007) [arXiv:0707.4181 [hep-th]].

14. L. Kofman, A. Linde and V. F. Mukhanov, "Inflationary theory and alternative cosmology," JHEP 0210, 057 (2002) [arXiv:hep-th/0206088].

15. R. Durrer, "Gauge Invariant Cosmological Perturbation Theory: A General Study And Its Application To The Texture Scenario Of Structure Formation," Fund. Cosmic Phys. 15, 209 (1994) [arXiv:astro-ph/9311041].

16. S. B. Giddings and A. Maharana, "Dynamics of warped compactifications and the shape of the warped landscape," Phys. Rev. D 73, 126003 (2006) [arXiv:hep-th/0507158].

17. K. Koyama, K. Koyama and F. Arroja, "On the 4D effective theory in warped compactifications with fluxes and branes," Phys. Lett. B 641, 81 (2006) [arXiv:hep-th/0607145]. 